\title{
Les tentatives d'organisation du culte musulman en France au prisme du principe de laïcité
}

Pierre-Henri Prélot

\section{OpenEdition}

12 Journals

Édition électronique

URL : http://journals.openedition.org/rdr/324

DOI : $10.4000 /$ rdr.324

ISSN : 2534-7462

Éditeur

Presses universitaires de Strasbourg

\section{Édition imprimée}

Date de publication : 6 novembre 2018

Pagination : 13-26

ISBN : 979-10-344-0023-2

ISSN : 2493-8637

Référence électronique

Pierre-Henri Prélot, «Les tentatives d'organisation du culte musulman en France au prisme du principe de laïcité ", Revue du droit des religions [En ligne], 6 | 2018, mis en ligne le 25 novembre 2019, consulté le 19 novembre 2020. URL : http://journals.openedition.org/rdr/324 ; DOI : https://doi.org/ $10.4000 /$ rdr.324

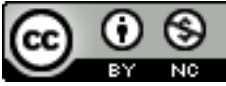

La revue du droit des religions est mise à disposition selon les termes de la Creative Commons Attribution - Pas d'Utilisation Commerciale 4.0 International - CC BY-NC 4.0. 


\section{LESTENTATIVESD'ORGANISATION DUCULTEMUSULMANENFRANCE AUPRISME DUPRINCIPE DELAḮITÉ}

\section{Pierre-Henri PRÉLOT}

Université de Cergy-Pontoise

\section{RÉSUMÉ}

Depuis une vingtaine d'années, les pouvoirs publics cherchent à organiser institutionnellement le culte musulman, afin d'en permettre la pratique paisible et de favoriser l'intégration des citoyens musulmans dans la communauté nationale. Cette politique n'est pas sans évoquer le régime des cultes reconnus tel qu'il fonctionnait au XIX ${ }^{e}$ siècle, et qui assurait à l'État un contrôle social sur les forces religieuses. Pour autant, les efforts d'organisation du culte musulman s'inscrivent aujourd'hui dans le cadre juridique de la laïcité, qui astreint les pouvoirs publics à une obligation de neutralité et leur impose de respecter l'autocompréhension des religions. L'objet de la présente réflexion est précisément de démontrer que ces efforts s'inscrivent en cohérence avec l'exigence de laïcité qui implique la garantie de la liberté de religion dans le respect de l'ordre public.

\section{Abstract}

For the past twenty years, the public authorities have sought to institutionally organize Islam in order to enable its peaceful practice and thereby promote the integration of Muslim citizens into the national community. This policy is not without evoking the regime of recognized religious bodies as it functioned in the nineteenth century, and which provided the State with social control over religious forces. However, efforts to organize the Muslim faith are now part of the legal framework of laïcité, which obliges the public authorities to be neutral and to respect the self-determination of religions. Our purpose is precisely to demonstrate that these efforts are consistent with the requirement of laïcité, which implies guaranteeing freedom of religion within the bounds of public order. 
$\mathrm{L}$ 'organisation du culte musulman en France est une préoccupation récurrente des pouvoirs publics, de droite comme de gauche, depuis le tournant des années 1990. La sédentarisation de la population immigrée dans un espace européen garant de la vie familiale et de la liberté religieuse a fait prendre progressivement conscience de la nécessité qu'il y avait de permettre aux musulmans, pour la plupart d'origine étrangère, de pratiquer convenablement leur religion, en mettant fin à ce que l'on a pu appeler l'«islam des caves». Dans le cas particulier de la France, où la communauté musulmane, régulièrement évaluée à 5 millions de membres, est la plus nombreuse d'Europe, ce sont également les affaires de voile à l'école, la fatwa lancée contre Salman Rushdie pour ses versets sataniques, ainsi que la montée du FIS en Algérie ${ }^{1}$, qui sont au point de départ, en 1989, de cette prise de conscience.

Malgré une convergence de principe des gouvernements de droite comme de gauche quant à la nécessité d'une politique volontariste, l'alternance accélérée des pouvoirs, particulièrement entre 1993 et 2002, a nui incontestablement à la cohérence de l'action publique. Bien qu'elle ait soulevé autant de difficultés nouvelles qu'elle n'en a résolu d'anciennes, la mise en place du Conseil français du culte musulman (CFCM), en 2003, a permis de tirer un trait sur une longue décennie de tergiversations quant au choix de l'interlocuteur musulman légitime, ce qui n'empêche pas les autorités de conserver des relations avec les grandes fédérations. Quoi qu'il en soit de ces divergences et de ces nuances, il est manifeste qu'au nombre des ministres de l'Intérieur qui depuis les années 1980 se sont montrés les plus actifs figurent des personnalités dont le parcours s'inscrit dans la plus pure tradition laïque - de Pierre Joxe à Bernard Cazeneuve, sans oublier Charles Pasqua et JeanPierre Chevènement - ce qui en soi signifie qu'à leurs yeux l'intégration de l'islam dans le cadre français n'a jamais été une entorse à cette tradition, mais au contraire le moyen d'y fondre la religion musulmane.

On assimile régulièrement le processus de création du Conseil français du culte musulman à la reprise en main autoritaire des cultes par Napoléon Bonaparte. Si le modèle centralisé du Conseil français du culte musulman n'est effectivement pas sans évoquer celui du Consistoire central israélite de France installé en 1808, il reste que le CFCM n'aurait pu être créé sans l'accord express des interlocuteurs musulmans engagés dans le processus de négociation, et que les conseils régionaux du culte musulman comme

1. On reprend ici les développements de Hakim El Karoui, dans son rapport pour l'Institut Montaigne de septembre 2016, Un islam français est possible. V. spéc. p. 91. 
le conseil national qui les fédère sont organisés sous une forme associative simple dont le fonctionnement est organiquement indépendant des autorités publiques. Et si l'organisation pyramidale du culte musulman reproduit dans ses formes l'idéaltype gallican qui prévalait sous le régime des cultes reconnus, c'est bien parce que cet idéaltype ne s'est pas dissous de lui-même avec la loi de 1905, mais qu'il est resté très prégnant dans le régime de séparation ${ }^{2}$. L'État français n'a jamais renoncé à dialoguer avec les religions, et pour cela il lui faut des interlocuteurs susceptibles de parler en leur nom afin de faire valoir leurs besoins propres, ce qui en aucune façon ne l'engage à les satisfaire au-delà de ce que requiert la garantie de la liberté de religion.

Si l'organisation du culte musulman par l'État désigne couramment la mise en place d'institutions représentatives, la création du CFCM n'est en réalité que le prélude à l'émergence d'un islam gallican, un islam de France intégré dans la vie sociale et respectueux des valeurs fondamentales qui structurent la société républicaine. Tel est en tout cas le projet des pouvoirs publics, qui cherchent moins à «reconnaître » l'islam au sens où on l'entendait au XIX ${ }^{e}$ siècle qu'à favoriser ses formes d'expression compatibles avec le projet libéral. Si donc l'on doit parler de reconnaissance, c'est d'abord et avant tout au sens d'une politique de reconnaissance telle que la définit Charles Taylor ${ }^{3}$, capable d'offrir aux citoyens musulmans les conditions de leur intégration à part entière dans une société française ouverte au pluralisme. Ce que l'on entend expliquer dans les développements qui suivent, c'est que ce projet de politique religieuse n'est en rien contraire aux exigences de la laïcité, mais qu'il s'inscrit au contraire dans la logique même du projet laique tel que le définit le titre premier de la loi de 1905, qui énonce les principes cardinaux de la liberté de conscience et du libre exercice des cultes (1), de la nonreconnaissance et de son corollaire la séparation financière (2) et qui inscrit l'exercice de la liberté religieuse dans les strictes limites de l'ordre public (3).

Pour faciliter, comme il entend le faire avec l'islam, l'intégration sociale d'une religion d'implantation récente, l'État est conduit à mobiliser le pouvoir normatif dont il dispose. Si le législateur n’a guère été sollicité que de façon indirecte jusqu'à présent ${ }^{4}$, en raison notamment du postulat d'intangibilité de

2. Pour une illustration et défense de ce modèle devant la Cour de Strasbourg, V. CEDH, Gde ch., 27 juin 2000, n² 27417/95, Cha'are Shalom Ve Tsedek c. France.

3. «La politique de reconnaissance», in Ch. TAYLOR, Multiculturalisme: différence et démocratie, Paris, Flammarion, 1992, p. 41-99.

4. La consolidation des baux emphytéotiques administratifs en vue de l'affectation à une association cultuelle d'un édifice du culte ouvert au public, dans la partie législative du Code général des collectivités territoriales (art. L. 1311-2), s'est opérée par voie d'ordonnance 
la loi de 1905, il dispose en revanche d'une importante marge de manœuvre pour décider par voie réglementaire dès lors que ne sont pas en cause les garanties fondamentales accordées aux citoyens pour l'exercice des libertés publiques $^{5}$. De surcroît, il existe en France une solide tradition administrative de recours aux circulaires et notes de service qui permet d'organiser les pratiques religieuses dans les marges du droit ${ }^{6}$. Mais quoi qu'il en soit, le simple exercice unilatéral du pouvoir normatif par les autorités étatiques est impuissant à ordonner les pratiques religieuses à lui seul, et la plupart du temps l'adoption d'un texte est l'aboutissement d'un processus de concertation destiné à faciliter son acceptation ultérieure par les pratiquants concernés. Un tel engagement des autorités religieuses envers les pouvoirs publics, qu'il est régulièrement demandé à ces dernières de consentir, ne prend évidemment toute sa signification qu'à travers un rituel d'officialisation solennelle, notamment sous la forme de déclarations publiques dans les médias ${ }^{7}$. Il peut également prendre la forme de chartes ou de proclamations écrites ${ }^{8}$ accueillies avec bienveillance par les pouvoirs publics, quand elles ne sont pas suscitées par eux de façon plus ou moins pressante ${ }^{9}$. C'est ce mélange complexe de droit classique et de soft law qui forme le maillage destiné à faciliter la pratique de l'islam dans les cadres où s'inscrit en France l'exercice des pratiques religieuses.

(art. 38 de la Constitution), ratifiée sans que la disposition elle-même ait fait l'objet d'un débat parlementaire.

5. À titre d'exemple, c'est un arrêté du 16 mars 2005 qui met en place une aumônerie militaire musulmane, et c'est un décret du 3 mai 2017 qui organise la formation civile et civique des aumôniers militaires, hospitaliers et pénitentiaires.

6. V. par ex. la circulaire ministérielle du 23 septembre 1967 relative aux autorisations d'absence pouvant être accordées aux fonctionnaires pour participation à des cérémonies religieuses, ou bien la circulaire du 19 février 2008 relative à la police des lieux de sépulture.

7. En 2009, le CFCM avait déclaré publiquement, sans approuver pour autant le principe d'une intervention législative, que le port du voile intégral n'est pas une prescription religieuse.

8. Charte du culte musulman en France, 1994; Déclaration d'intention relative aux droits et obligations des fidèles du culte musulman, 1999; Convention citoyenne des musulmans de France pour le vivre-ensemble, 2014; Proclamation de l'islam en France et Charte de l'imam, 2017.

9. On se souvient que Napoléon avait réuni en 1806 une assemblée des notables juifs, puis en 1807 un Grand Sanhédrin, afin d'obtenir d'eux des engagements précis visant au respect des lois civiles. Aujourd'hui, l'intervention des pouvoirs publics est évidemment plus respectueuse de l'indépendance des autorités religieuses. 


\section{LIBERTÉ DE CONSCIENCE ET LIBRE EXERCICE DES CULTES}

\subsection{LA LIBERTÉ DE CONSCIENCE}

Les efforts de l'État en vue de l'intégration de l'islam sont généralement justifiés, et à juste titre, du point de vue du libre exercice des cultes, que la République "garantit», selon la formule de l'article $1^{\text {er }}$ de la loi de 1905. Mais cette garantie du libre exercice des cultes comprend un préalable, qui est celui du respect de la liberté de conscience, que la République "assure » selon ce même article. Assurer la liberté de conscience, c'est évidemment faire en sorte que l'État ne la viole pas lui-même, mais c'est également la protéger contre les atteintes des tiers. De surcroît, alors que la liberté de religion présente une forte dimension collective et organisée, la liberté de conscience est purement individuelle, et elle peut être aussi bien religieuse qu'a- ou antireligieuse, en sorte qu'elle doit être protégée y compris contre les religions elles-mêmes, qui ne détiennent pas en dépôt perpétuel la conscience de leurs adeptes. Ce principe fondamental d'autonomie de la conscience individuelle constitue un point de crispation récurrent dans les discussions avec les associations musulmanes les plus rigoristes. La première version de la Déclaration d'intention relative aux droits et obligations des fidèles du culte musulman, que devaient signer les participants au processus de concertation (Istichâra) instauré par Jean-Pierre Chevènement en 1999, comprenait ainsi une mention explicite du droit à changer de religion, finalement retirée du texte soumis à la signature le 28 janvier 2000 à la demande de l'Union des organisations islamiques de France (UOIF). L'incident est révélateur des tensions que suscite chez les autorités religieuses musulmanes cette question de l'apostasie. Si les pouvoirs publics ont depuis lors renoncé à exiger cet engagement explicite que leurs interlocuteurs musulmans avaient perçu comme une humiliation, l'exigence de respect de la liberté de conscience tend à réapparaître aujourd'hui, encore que de façon plutôt timide, sous la forme d'un engagement volontaire dans les chartes élaborées par les représentants de l'islam ${ }^{10}$.

10. La Convention citoyenne des musulmans de France pour le vivre-ensemble de 2014 rappelle en son article $1^{\text {er }}$ que «la France assure à tous les citoyens la liberté de croire ou de ne pas croire, de pratiquer ou de ne pas pratiquer une religion». L'article 6 énonce quant à lui que «tous les cultes, toutes les croyances et toutes les personnes quelle que soit leur origine ethnique ou religieuse vivent libres et respectées en France». Plus explicite, la Charte de l'imam de France, adoptée en mars 2017 par le CFCM, énonce (3०) «que Dieu qui a créé les hommes et les femmes égaux et dignes, leur a donné la liberté de choisir leurs voies et leurs convictions à l'abri de toute contrainte. Aussi, l'imam appelle à ce que tout citoyen puisse être respecté pour ce qu'il est et pour ce qu'il croit: "Nulle 


\subsection{LE LIBRE EXERCICE DES CULTES}

Si les pouvoirs publics attachent tant de prix à la structuration de l'islam dans le cadre national, c'est principalement au nom du libre exercice des cultes qu'ils le justifient. Les motifs d'ordre public qui orientent leur action dans les faits ne sont jamais en ce sens que des «restrictions justifiées » au sens où l'entend l'article $1^{\text {er }}$ de la loi de 1905. De fait, la République est non seulement tenue de ne pas entraver le libre exercice des cultes, mais l'obligation qui lui incombe de le garantir implique de surcroît une intervention active des pouvoirs publics afin de lui donner toute son effectivité. Il en va de la liberté de religion comme de toutes les libertés publiques: il ne suffit plus aujourd'hui de les proclamer, mais elles doivent faire l'objet des aménagements indispensables à leur exercice.

Concrètement, l'intervention des pouvoirs publics a vocation à protéger l'ensemble des domaines de l'activité religieuse qui relèvent du «culte», sans pour autant remettre en cause le partage historique qui s'est établi en France entre les champs de compétence étatique et religieuse, particulièrement dans les matières sensibles touchant à l'état et au statut des personnes, qui relèvent, depuis la Révolution, de la compétence exclusive de l'État (mariage, filiation, successions notamment). L'intervention des pouvoirs publics pourra ainsi porter sur des questions telles que la pratique du culte proprement dit $^{11}$, les fêtes religieuses, le respect des prescriptions alimentaires ${ }^{12}$, les rites funéraires ${ }^{13}$, la transmission de la foi (catéchèse), autant de pratiques qui caractérisent l'exercice d'un culte compris dans son sens le plus étendu. On ne présentera pas dans le détail l'ensemble des dispositions mises en œuvre depuis une vingtaine d'années par les pouvoirs publics, et qui sont dans l'ensemble bien connues. En revanche, ce qui mérite d'être souligné, c'est que la garantie du libre exercice des cultes ne relève pas du seul gouvernement et de l'administration d'État placée sous son autorité. Elle relève au moins autant des collectivités territoriales et en particulier des municipalités, auxquelles il incombe de donner toute

contrainte dans la religion" (Coran, 2: 256)». Ce texte, dont les mosquées devaient être invitées à faire de la signature «un élément essentiel à prendre en compte lors du recrutement d'un imam», a été vivement contesté par la plupart des fédérations représentées au CFCM (CIMG, CCMTF, FNGMP, UOIF, Foi et Pratique).

11. Permettre aux musulmans de disposer de lieux de culte en nombre suffisant, encourager la formation d'imams intégrés dans la culture nationale, garantir le respect des grandes fêtes religieuses, organiser l'aumônerie dans les services publics...

12. Organisation de l'abattage rituel, mise à disposition de menus de substitution dans les cantines.

13. Création d'espaces confessionnels dans les cimetières. 
son effectivité à la politique religieuse que le gouvernement entend conduire. Ce qui mérite également d'être souligné, c'est que la politique religieuse des pouvoirs publics fait l'objet de la part du juge, administratif mais aussi judiciaire, d'un accompagnement jurisprudentiel qui n'est jamais que la garantie juridictionnelle de l'effectivité du libre exercice des cultes.

\section{NON-RECONNAISSANCE ET NON-FINANCEMENT DES CULTES}

\subsection{LA SÉPARATION ORGANIQUE (NON-RECONNAISSANCE)}

L'organisation par la puissance publique du culte musulman s'apparente ainsi qu'on a pu le dire en introduction à une forme de reconnaissance officielle de l'islam, qui paraît de prime abord remettre en cause la règle d'or de la loi de 1905 selon laquelle «la République ne reconnaît [...] aucun culte » (art. 2). De fait, si la République a cessé en 1905 de les reconnaître, c'est au sens où la loi est venue mettre fin au régime dit des cultes reconnus qui les organisait sous la forme d'établissements publics dotés de statuts différenciés. Dans le nouveau régime de séparation en revanche, ils sont organisés sous la forme identique d'associations (cultuelles) des lois de 1901-1905. La non-reconnaissance s'identifie ainsi à une égalité de toutes les religions devant la loi commune, et à l'absence de distinction statutaire entre celles qui seraient reconnues et d'autres qui ne le seraient pas. Cette organisation générale du régime des cultes n'est pas remise en cause par le processus d'intégration de l'islam.

Mais de ce postulat d'égalité des religions devant la loi il ne résulte pas pour autant que celle-ci ne doive opérer aucune distinction entre elles. Chacune des religions établies en France est tributaire d'une histoire particulière qui détermine son ancrage dans la communauté nationale, et dont on retrouve toujours plus ou moins l'imprégnation dans le système de droit. C'est ainsi par exemple que la loi de 1905 a dû régler le sort du patrimoine religieux, et en particulier des édifices du culte, tous catholiques, nationalisés à la Révolution, mais également celui des édifices construits au XIX ${ }^{e}$ siècle, en fonction de l'identité de leur propriétaire (la commune ou l'établissement public du culte). Ces dispositions ne concernent jamais que les anciens cultes reconnus, et en particulier l'Église catholique, en sorte que la loi de 1905 organise de facto un régime de droit différencié qui ne concerne pas nécessairement toutes les religions. Compte tenu de la longue histoire coloniale de la France, compte tenu du nombre important de musulmans aujourd'hui établis en métropole, compte tenu enfin du caractère récent de cette implantation, il n'y a donc 
rien d'étonnant à ce que des dispositions tenant compte de cette situation particulière puissent être adoptées en tant que de besoin afin de favoriser leur pleine intégration dans la communauté nationale ${ }^{14}$. De surcroît, toutes les religions présentent un certain nombre de spécificités ${ }^{15}$ qui font qu'aucune n'est identique à une autre, et qui sont de nature à justifier un aménagement juridique particulier de la liberté de religion ${ }^{16}$. La juste application du principe d'égalité implique que le droit prenne en considération les différences de situation, a fortiori lorsqu'est en cause l'exercice d'une liberté fondamentale telle que la liberté de religion. Il n'est donc nullement besoin, comme on le fait souvent, d'invoquer la notion d'inégalité de compensation ou de discrimination positive pour expliquer les aménagements destinés à permettre la pratique de leur culte par les musulmans.

\subsection{LA SÉPARATION FINANCIÈRE}

La décision QPC du 21 février 2013, Association pour la promotion et l'expansion de la laïcitée ${ }^{17}$, est venue mettre fin à un débat ancien concernant le financement public des cultes, en indiquant que si la Constitution prohibe le fait de salarier un culte, en revanche l'interdiction de leur subventionnement prend sa source dans la loi de 1905 et elle seule, en sorte que rien n'interdit au législateur de s'en affranchir. Le décret du 3 mai 2017 relatif aux aumôniers militaires, hospitaliers et pénitentiaires et à leur formation civile et civique, dont l'objet premier est de permettre le recrutement d'aumôniers musulmans formés aux valeurs de la République et rémunérés en contrepartie, en particulier des aumôniers pénitentiaires, trouve ainsi sa justification dans l'article 2 de la loi de 1905 elle-même, qui excepte de la prohibition principielle «les dépenses relatives à des services d'aumônerie». De la même façon, les baux emphytéotiques administratifs (BEA) consentis par les communes aux associations cultuelles musulmanes en vue de permettre la construction

14. La circulaire du 19 février 2008 relative à la police des lieux de sépulture, ou encore le décret du 3 mai 2017 relatif aux aumôniers militaires, hospitaliers et pénitentiaires et à leur formation civile et civique, bien que rédigés en termes généraux, ont été adoptés en considération de nécessités propres à la seule religion musulmane.

15. V. sur ce point P.-H. PréLot, «Les religions et l'égalité en droit français», RDP 2001, p. $737-775$.

16. Il existe ainsi des prescriptions alimentaires et des prescriptions funéraires qui sont particulières à l'islam. De même, la vie monastique est propre au catholicisme (et au bouddhisme), en sorte qu'historiquement le régime juridique des congrégations religieuses a été conçu pour la seule Église catholique, même s'il a été étendu par le Conseil d'État aux autres religions depuis les années 1970.

17. Cons. const., 21 févr. 2013, n 2012-297 QPC. 
de mosquées ont leur fondement dans la partie législative du Code général des collectivités territoriales qui déroge à l'interdiction générale de l'article 2 .

Mais en dehors de cette dérogation qui est d'interprétation stricte ${ }^{18}$, le financement du culte proprement dit reste purement privé, et c'est ce qui explique que dans le cadre de l'Instance de dialogue avec l'islam de France, mise en place au lendemain de l'attentat contre Charlie Hebdo en janvier 2015, les pouvoirs publics aient suggéré la création d'une association cultuelle nationale musulmane, indépendante des pouvoirs publics, chargée de pourvoir aux besoins de la pratique religieuse. Sa mise en place, compte tenu de la rivalité des fédérations, reste néanmoins très aléatoire. Quant à la Fondation de l'islam de France ${ }^{19}$, elle a pour objet de financer des projets dépourvus d'objet cultuel dans le domaine de l'éducation et de la culture.

Outre qu'elle entérine le recours à la loi afin de permettre un financement public des activités religieuses, la décision QPC du 21 février 2013 a pour effet de conforter la jurisprudence du Conseil d'État validant le financement par les collectivités territoriales de projets en rapport avec des édifices religieux ou des pratiques cultuelles, dès lors que les décisions de financement « répondent à un intérêt public local». C'est ainsi que dans trois décisions du 19 juillet 2011, le Conseil d'État a pu confirmer le financement, par une communauté urbaine, d'un abattoir destiné aux abattages rituels à l'occasion de l'Aïd el Kebir $^{20}$, la mise à disposition pérenne et exclusive d'une salle polyvalente en vue de son utilisation par une association cultuelle musulmane ${ }^{21}$, ainsi que la conclusion d'un bail emphytéotique administratif entre une commune et une association cultuelle en vue de la construction d'une mosquée sur un terrain communal mis à disposition contre un loyer symbolique d'un euro annuel $^{22}$. L'assouplissement de la jurisprudence administrative en matière de financement public des cultes permet ainsi d'accompagner l'action des collectivités territoriales en vue de faciliter la pratique de l'islam. On peut d'ailleurs voir là l'explication principale de cet assouplissement, dont l'origine première remonte à un arrêt de 2005 relatif à la construction d'un presbytère dans un territoire de l'outre-mer ${ }^{23}$.

18. V. CE, 10 févr. 2017, n 395433, Ville de Paris, jugeant que l'article L. 1311-2 CGCT a «dérogé» à l'interdiction générale de l'article 2 , et que l'interprétation stricte de cette dérogation excluait que des BEA à objet religieux puissent être conclus en faveur d'associations de la loi de 1901.

19. Créée par un décret du 5 décembre 2016, elle est reconnue d'utilité publique.

20. CE, 19 juill. 2011, n 309161, Communauté urbaine du Mans - Le Mans Métropole.

21. CE, 19 juill. 2011, $\mathrm{n}^{\circ} 313518$, Commune de Montpellier.

22. CE, 19 juill. 2011, n 320796, Mme V.

23. CE, 16 mars 2005, $\mathrm{n}^{\circ}$ 265560, Ministre de l'Outre-mer. 


\section{LE RESPECT DE L'ORDRE PUBLIC}

C'est l'ordre public qui définit au niveau constitutionnel ${ }^{24}$ comme au niveau législatif ${ }^{25}$ les «limites » dans lesquelles s'inscrit en France l'exercice de la liberté religieuse. Si aux yeux des pouvoirs publics, son respect est la condition du soutien accordé aux pratiques religieuses (3.1), cette notion tend aujourd'hui à se reconfigurer en vue de restreindre la visibilité de l'islam dans l'espace public (3.2).

\subsection{LE RESPECT DE L'ORDRE PUBLIC COMME CONDITION DU SOUTIEN À L'ISLAM}

La nécessité du dialogue avec les représentants de l'islam ne se fait jamais plus ressentir qu'au lendemain d'attentats terroristes. C'est ainsi que la mise en place d'une Instance de dialogue avec l'islam de France, en juin 2015, est une réponse directe aux attentats de janvier contre Charlie Hebdo et l'Hyper Casher de la Porte de Vincennes. Bernard Cazeneuve pouvait ainsi affirmer le $1^{\text {er }}$ juillet 2015, devant les responsables musulmans réunis pour la rupture du jeûne de Ramadan, que «le meilleur rempart contre le terrorisme, ce sont les musulmans de France eux-mêmes et la République rassemblée dans toutes ses compétences [...], rassemblant tous ses enfants, d'où qu'ils viennent, autour de ses valeurs». Favoriser l'encadrement religieux des musulmans pratiquants par des personnes formées à la laiicité et aux valeurs de la République, constitue aux yeux des pouvoirs publics le meilleur sinon le seul moyen d'entraver sur le long terme le radicalisme religieux qui se nourrit de l'ignorance et de l'endoctrinement. C'est également permettre d'intégrer pleinement dans la communauté nationale des personnes aux origines majoritairement étrangères, et qui vivent au quotidien le rejet et l'exclusion sociale ${ }^{26}$. Il s'agit enfin, à travers le soutien explicite apporté aux musulmans qui ne demandent qu'à se fondre dans la communauté nationale, d'isoler les minorités radicalisées et de combattre l'amalgame courant qui confond islam et violence, et tient cette religion pour incompatible avec les valeurs occidentales de démocratie,

24. Art. 10 de la Déclaration des droits de l'homme et du citoyen: «Nul ne doit être inquiété pour ses opinions, même religieuses, pourvu que leur manifestation ne trouble pas l'ordre public établi par la loi.»

25. L. 19 déc. 1905, art. 1: «La République assure la liberté de conscience. Elle garantit le libre exercice des cultes sous les seules restrictions édictées ci-après dans l'intérêt de l'ordre public. »

26. C'est d'ailleurs pour cette raison que la politique d'intégration républicaine de l'islam est souvent comprise comme une forme de discrimination positive en faveur de minorités défavorisées. 
de liberté ou encore d'égalité des sexes. La condamnation, par les représentants en vue de l'islam, des violences terroristes et des pratiques religieuses extrêmes, ainsi que les manifestations publiques de loyauté à la République et aux institutions, quelles que soient leurs formes, sont pour cette raison particulièrement attendues des pouvoirs publics, comme contrepartie de leur soutien à la communauté musulmane. C'est d'ailleurs la raison d'être principale de ces chartes suscitées par les musulmans eux-mêmes, que d'attester solennellement de la sincérité de leur engagement républicain. Il reste que ces attentes ne manquent pas de nourrir à rebours un sentiment de défiance et d'instrumentalisation par les pouvoirs publics, soupçonnés de vouloir rétablir à travers un soutien officiel de l'islam une religion administrée, une religion sous tutelle ${ }^{27}$, qui fait ressurgir le spectre des anciens rapports de domination coloniale. Quant aux pouvoirs publics et à l'opinion, ils sont enclins à voir dans les positionnements ambigus de certains représentants musulmans une marque de défiance et de déloyauté. On retrouve là toutes les difficultés du dialogue entre les pouvoirs publics et les représentants de l'islam, qu'accentuent la grande diversité de la population musulmane et les conflits d'obédience qui la fracturent encore plus. Ces difficultés ne sont en rien propres à la France, et elles existent dans les autres pays européens qui ont choisi la voie du dialogue avec l'islam, ce qui permet de conclure que le cadre laïque français n'est pas particulièrement en cause.

La politique de bienveillance envers l'islam se déploie ainsi en miroir des mesures de protection de l'ordre public, et en particulier de celles adoptées en matière de lutte contre le terrorisme. Peuvent être mentionnées ici la loi du 13 novembre $2014^{28}$, qui crée une interdiction de sortie du territoire, visant à empêcher les islamistes radicalisés de rejoindre le djihad, ou encore la loi du 21 juillet $2016^{29}$ relative à l'état d'urgence, qui autorise le ministre de l'Intérieur et les préfets à ordonner la fermeture provisoire des lieux de culte au sein desquels sont tenus des propos constituant une provocation à la haine ou à la violence ou une provocation à la commission d'actes de terrorisme ou faisant l'apologie de tels actes. La loi du 30 octobre $2017^{30}$

27. La nomination, à la tête de la Fondation pour l'islam de France, d'un ancien ministre de l'Intérieur, aux convictions laïques et gallicanes bien ancrées, en lieu et place d'une personnalité de confession musulmane, a été perçue comme une marque de défiance du gouvernement.

28. L. n 2014-1353, 13 nov. 2014, renforçant les dispositions relatives à la lutte contre le terrorisme.

29. L. $\mathrm{n}^{\circ}$ 2016-987, 21 juill. 2016, prorogeant l'application de la loi $\mathrm{n}^{\circ}$ 55-385 du 3 avril 1955 relative à l'état d'urgence et portant mesures de renforcement de la lutte antiterroriste.

30. L. $n^{\circ}$ 2017-1510, 30 oct. 2017, renforçant la sécurité intérieure et la lutte contre le terrorisme. 
renforçant la sécurité intérieure et la lutte contre le terrorisme pérennise ces dispositions, en ajoutant comme motif nouveau de fermeture provisoire (six mois) «les idées ou théories qui sont diffusées». Un tel énoncé, qui a été maintenu dans la loi en dépit de son caractère flou, accrédite le postulat d'une porosité entre islamisme radical et terrorisme. Quoi qu'il en soit, le contrôle exercé par le juge administratif permet théoriquement de vérifier que les décisions de fermeture ne remettent pas en cause la liberté religieuse des autres adeptes ${ }^{31}$.

\subsection{LA RECONFIGURATION DE L'ORDRE PUBLIC}

Dans les débats de l'Assemblée nationale constituante relatifs à l'article 10 de la Déclaration des droits de l'homme et du citoyen, Mirabeau qui défendait la liberté de religion avait esquissé une définition «matérielle» de l'ordre public qui préfigure celle qu'en donnera par la suite la doctrine administrative de la Troisième République. Il s'agissait alors d'empêcher qu'au nom de la protection de l'ordre public l'exercice du culte soit restreint pour les protestants et les juifs.

«Ce qui est de la police, expliquait-il, c'est d'empêcher que personne ne trouble l'ordre et la tranquillité publique. Voilà pourquoi elle veille dans vos rues, dans vos places, autour de vos maisons, autour de vos temples; mais elle ne se mêle point de régler ce que vous y faites; tout son pouvoir consiste à empêcher que ce que vous y faites ne nuise à vos concitoyens.

Je trouve donc absurde encore de prétendre que, pour prévenir le désordre qui pourrait naître de vos actions, il faut défendre vos actions: assurément cela est très expéditif; mais il m'est permis de douter que personne ait ce droit $^{32} »$.

L'article $1^{\text {er }}$ de la loi de 1905 érigeant lui aussi l'ordre public en «limite» au libre exercice des cultes, c'est le juriste protestant Alexandre Ribot qui à son tour dénoncera lors des débats parlementaires de 1905 la conception très instrumentale que s'en font les auteurs de la loi: «Je voudrais bien savoir ce que c'est que l'ordre public. On le fait servir pour justifier un texte, pour se tirer d'embarras ${ }^{33} »$. Aujourd'hui, c'est encore cette notion controversée de

31. V. par ex. CE, ord., 6 déc. 2016, n 405476. Le juge vérifie qu'en cas de fermeture administrative d'une mosquée, il existe des lieux de culte à proximité permettant de garantir la liberté de culte des fidèles.

32. Séance du dimanche 23 août 1789: Moniteur universel, p. 376.

33. $2^{\mathrm{e}}$ séance du 15 juin 1905: JO débats Chambre des députés, p. 2253. 
l'ordre public qui est au cœur des débats relatifs à l'islam, et à sa visibilité sociale. C'est ainsi qu'en 2003, le rapport Stasi expliquait, pour recommander l'adoption d'une loi interdisant le port des signes religieux ostensibles dans les écoles publiques, qu'«aujourd'hui la question n'est plus la liberté de conscience, mais l'ordre public» remis en cause par «les tensions et les affrontements dans les établissements autour de questions religieuses ${ }^{34} »$. Quant à la loi du 11 octobre 2010 interdisant la dissimulation du visage dans l'espace public, et dont l'objet est la prohibition du port du voile intégral par les femmes musulmanes, elle trouve également son fondement dans l'ordre public, entendu au sens immatériel des «exigences fondamentales du "vivre ensemble" dans la société française ${ }^{35}$ \%.

L'organisation du culte musulman s'opère ainsi dans un contexte général de redéfinition des conditions de l'expression religieuse dans l'espace public, qui affecte principalement l'islam même si les lois encadrant le port de signes et de tenues vestimentaires ont une portée qui se veut générale. Et si de telles lois n'ont pas pour fondement explicite la laïcité, il n'en reste pas moins qu'elles ont pour objet de limiter une expression religieuse au nom de l'ordre public, en sorte qu'elles s'inscrivent de facto dans l'orbite de l'article $1^{\text {er }}$ de la loi de 1905, matrice de la laïcité républicaine dont la substance se trouve ainsi profondément redéfinie. On sait en effet qu'à l'époque, le législateur républicain avait été attentif à ne pas restreindre l'expression des religions dans l'espace public, qu'il s'agisse du port de signes et tenues religieuses ou des cérémonies religieuses dans l'espace public (processions notamment) ${ }^{36}$. Cette «nouvelle laïcité ${ }^{37} »$, que le juge est chargé d'endiguer dans les limites qu'autorise le respect de la liberté d'expression religieuse ${ }^{38}$, tend à nourrir une défiance mutuelle des interlocuteurs publics et religieux, dont chacun met en doute la sincérité de l'autre.

34. Rapport de la Commission de réflexion sur l'application du principe de laïcité dans la République, déc. 2003, p. 58.

35. Exposé des motifs de la loi du 11 octobre 2010.

36. V. sur cette question notre article: «L'interdiction de la dissimulation du visage à la lumière du principe de laïcité»: Revue du droit des religions, ${ }^{\circ} 2$, nov. 2016, p. 11-27.

37. V. S. Hennette VAuchez et V. Valentin, L'affaire Baby-loup ou la nouvelle laïcité, Paris, LGDJ, 2014.

38. Cass. soc., 22 nov. 2017, n 13-19.855, Bougnaoui, à propos du port du foulard islamique dans l'entreprise; CE, ord., 26 août 2016, n' 402742, Ligue des droits de l'homme et autres, à propos des arrêtés portant interdiction du burkini sur les plages; CE, avis, 23 déc. 2013 (non publié), à propos du port de signes religieux par les accompagnatrices scolaires. 
On mesure dans les développements qui précèdent toute la complexité et toutes les difficultés d'un processus dont le succès s'inscrit nécessairement dans le temps long. Si un tel processus paraît contredire l'idéal républicain de laïcité, c'est moins pour des raisons juridiques que parce qu'il reconnaît aux religions un rôle de premier plan dans la structuration de la vie sociale. L'État a cessé désormais d'incarner un idéal d'émancipation collective dont le projet laique avait pu être le vecteur sous la Troisième République, et c'est pourquoi il est de plus en plus conduit à prendre appui, ainsi que l'explique remarquablement Philippe Portier ${ }^{39}$, sur les forces de la société civile, et notamment sur les forces religieuses, pour retisser un lien social dont il ne parvient plus à assurer à lui seul la cohésion. Les religions ont joué pendant la période récente un rôle précieux d'apaisement des tensions intercommunautaires, auquel les pouvoirs publics n'ont pas manqué d'être sensibles, particulièrement à la suite de l'attentat de Saint-Étienne-du-Rouvray, et il est vraisemblable que le nouveau président de la République, s'il entend lui imprimer sa marque personnelle, ne remettra pas fondamentalement en cause cette politique de reconnaissance de l'islam dans le but de renforcer l'unité nationale et la cohésion sociale.

39. L'État et les religions en France. Une sociologie historique de la laïcité, Presses universitaires de Rennes, 2016. V. en particulier la troisième partie. - V. également "Comment les religions ont-elles contribué à l'unité nationale?», La Croix, 8 janv. 2018. 